\title{
A Counterexample to Fishburn's Conjecture on Finite Linear Qualitative Probability
}

\author{
Marston Conder ${ }^{1}$ Arkadii Slinko * \\ Department of Mathematics, The University of Auckland, Private Bag 92019, \\ Auckland, NZ
}

\begin{abstract}
Kraft, Pratt and Seidenberg (1959) provided an infinite set of axioms which, when taken together with de Finetti's axiom, gives a necessary and sufficient set of "cancellation" conditions for representability of an ordering relation on subsets of a set by an order-preserving probability measure. Fishburn (1996) defined $f(n)$ to be the smallest positive integer $k$ such that every comparative probability ordering on an $n$-element set which satisfies the cancellation conditions $C_{4}, \ldots, C_{k}$ is representable. By the work of Kraft et al. (1959) and Fishburn $(1996,1997)$, it is known that $n-1 \leq f(n) \leq n+1$ for all $n \geq 5$. Also Fishburn proved that $f(5)=4$, and conjectured that $f(n)=n-1$ for all $n \geq 5$. In this paper we confirm that $f(6)=5$, but give counter-examples to Fishburn's conjecture for $n=7$, showing that $f(7) \geq 7$. We summarise, correct and extend many of the known results on this topic, including the notion of "almost representability", and offer an amended version of Fishburn's conjecture.
\end{abstract}

Key words: comparative probability, cancellation conditions, discrete cones, Fishburn's conjecture

\section{Introduction}

The topic of this article dates back to fundamental work by Bruno de Finetti (1931), who asked whether a certain axiom for a comparative probability ordering relation $\succeq$ on the subsets of a set is sufficient for the existence of an

\footnotetext{
* Corresponding author.

Email addresses: conder@math.auckland.ac.nz (Marston Conder), slinko@math. auckland.ac.nz (Arkadii Slinko).

1 Research supported by N.Z. Centres of Research Excellence Fund (grant UOA 201)
} 
order-preserving probability measure on that set. For infinite sets, assuming there is a uniform partition into an arbitrary number of events, Savage (1954) answered this question positively. For finite sets of cardinality five or more, a negative answer to this question was given by Kraft et al. (1959). It became clear from these observations that comparative probability is a broader concept than probability.

Nevertheless, de Finetti's axioms were very attractive, especially for use in applications where relative frequency characterization of probabilities is not available or meaningful, for example when we have to deal with the so-called "subjective probability" (also called "intuitive probability" or "personal probability"), which is essentially a belief of an individual that some events are more probable than the others (Luce \& Suppes, 1965). This led to development of comparative probability as a separate area of research (see a recent survey of Giuliana Regoli (2000)).

Sometimes comparative probability orderings emerge in circumstances where we cannot speak even about subjective probability. For example, they appeared in an article by Vladimir Danilov (1987), who, in the case of dichotomous preferences, characterised all social choice rules which satisfy the famous Arrow's condition of Independence of Irrelevant Alternatives. It appeared that each such rule corresponds to a comparative probability ordering, and the rule is a weighted majority rule if and only if the comparative probability ordering is representable by an order-preserving probability measure on the set of voters.

Kraft et al. (1959) discovered an infinite set of axioms which, when taken together with de Finetti's axiom, gives a necessary and sufficient set of conditions for representability of an ordering relation on subsets by an order-preserving probability measure. Implicitly, these axioms involved a concept of a multiset, which was not widely understood at the time, and reformulation of these axioms by Scott (1964) was predominantly used. Krantz, Luce, Suppes and Tversky (1971) called these axioms cancellation conditions. Fishburn (1996, 1997) opined that they "play a central role in the representational theory of measurement", and devoted much effort to their study.

Fishburn (1996) introduced a function $f(n)$, which, for each positive integer $n$, measures the maximal deviation of a comparative probability ordering on an $n$-element set from one which arises from an order-preserving probability measure. It follows from Kraft et al. (1959) that $f(n) \leq n+1$. Fishburn (1997) then proved that $f(n) \geq n-1$ for all $n \geq 5$, and so combining these two results gives the following bounds for this function: $n-1 \leq f(n) \leq n+1$. Fishburn also proved that $f(5)=4$, and conjectured that $f(n)=n-1$ for all $n \geq 5$.

In this paper we confirm that $f(6)=5$, but give counter-examples to Fish- 
burn's conjecture for $n=7$ showing that $f(7) \geq 7$. We summarise all known results, correcting and extending some of them, and offer an amended version of Fishburn's conjecture. In doing this, we consider the concept of an "almost representable" comparative probability ordering, and develop a construction method using this as a tool for finding lower bounds for $f(n)$.

\section{Preliminaries}

To formulate cancellation conditions, let us first recall some other definitions and fix some notation. Here $2^{X}$ denotes the set of all subsets of a set $X$.

Definition 1 Let $X$ be a finite set. Any reflexive, complete and transitive binary relation $\succeq$ on $2^{X}$ will be called an order on $2^{X}$. (Note: sometimes this is called a complete pre-order or a weak order.) Such an order $\succeq$ gives rise to two other relations $\succ$ and $\sim$ on $2^{X}$, defined for all $x, y \in X$ by

(a) $x \succ y \Longleftrightarrow x \succeq y$ and $\operatorname{not}(y \succeq x)$;

(b) $x \sim y \Longleftrightarrow x \succeq y$ and $y \succeq x$.

If an order $\succeq$ is anti-symmetric, then all equivalence classes (under the relation $\sim)$ are singletons, and $\succeq$ is called a linear order.

Definition 2 Let $X$ be a finite set. A linear order $\succeq$ on $2^{X}$ is called a comparative probability ordering on $X$, if $A \succ \emptyset$ for every non-empty subset $A$ of $X$, and $\succeq$ satisfies de Finetti's axiom, namely for all $A, B, C \in 2^{X}$,

$$
A \succeq B \Longleftrightarrow A \cup C \succeq B \cup C \text { whenever }(A \cup B) \cap C=\emptyset .
$$

For convenience, we will further suppose that $X=I_{n}=\{1,2, \ldots, n\}$. Note that if we have a probability measure $\mathbf{p}=\left(p_{1}, \ldots, p_{n}\right)$ on $X$, where $p_{i}$ is the probability of $i$, then we know the probability of every event $A$, by the rule $p(A)=\sum_{i \in A} p_{i}$. We may now define a relation $\succeq$ on $2^{X}$ by

$$
A \succeq B \quad \text { if and only if } \quad p(A) \geq p(B) .
$$

If $p_{i}>0$ for all $i$, and the probabilities of all events are different, then $\succeq$ is a comparative probability ordering on $X$, and any such ordering is called representable (e.g. Regoli, 2000). On the other hand, any comparative probability ordering $\succeq$ which is not obtainable in this way is called non-representable.

It can also be possible that for some measure $\mathbf{p}$ on $X$ the probabilities of two or more events coincide, that is $p(A)=p(B)$ when $A \succeq B$ but $A \neq B$. In this case we have an ordering $\succeq$ which still satisfies the de Finetti axiom (1), and we call it a weak comparative probability ordering arising from $\mathbf{p}$. 
If we have a non-representable comparative probability ordering $\succeq$ on $X$, then we cannot specify the exact probability of every event, but if $A \succ B$ then we can say that $A$ is more probable than $B$. The class of comparative probability measures is therefore broader than the class of probability measures (Kraft et al. (1959)). A non-representable comparative probability ordering $\succeq$ on $X$ is said to almost agree with the measure $\mathbf{p}$ on $X$ if

$$
A \succeq B \Longrightarrow p(A) \geq p(B)
$$

If such a measure $\mathbf{p}$ exists, then the ordering $\succeq$ is said to be almost representable. When $\mathbf{p}$ defines a weak comparative probability ordering on $X$, the ordering $\succeq$ might not be representable.

We now recall that a set is a special case of a multiset. Multisets are collections of objects which may include several copies of the same object. If we consider multisets on $X$, then we allow an element $x \in X$ to enter such a multiset $M$ with multiplicity $\mu_{M}(x)$, which is a non-negative integer. Multiset union $U$ is an operation over multisets that adds multiplicities of elements, that is, $\mu_{A \cup B}(x)=\mu_{A}(x)+\mu_{B}(x)$. See Stanley (1997) for more information on multisets.

Definition 3 A linear order $\succeq$ on $2^{X}$ is said to satisfy the mth cancellation condition $C_{m}$ if for no $m$ distinct comparisons $A_{i} \succ B_{i}$ of subsets (for $1 \leq$ $i \leq m)$, there exist positive integers $a_{1}, \ldots, a_{m}$ such that the following two multiset unions coincide:

$$
\bigcup_{i=1}^{m} \underbrace{\left(A_{i} \cup \ldots \cup A_{i}\right)}_{a_{i}}=\bigcup_{i=1}^{m} \underbrace{\left(B_{i} \cup \ldots \cup B_{i}\right)}_{a_{i}} .
$$

Alternatively, in the spirit of Scott (1964) (and also Suppes (1974)), condition (3) can be reformulated in terms of characteristic functions of subsets, as follows:

$$
\sum_{i=1}^{m} a_{i} \chi_{A_{i}}=\sum_{i=1}^{m} a_{i} \chi_{B_{i}}
$$

where the characteristic function $\chi_{S}$ of the subset $S \subset X$ is given by $\chi_{S}(x)=1$ if $x \in S$ and $\chi_{S}(x)=0$ if $x \notin S$.

Example 4 The non-representable comparative probability ordering $\succeq$ on $2^{I_{5}}$ constructed in Kraft et al. (1959) does not satisfy the condition $C_{4}$ since it contains the following comparisons:

$$
\{1,3\} \succ\{2,4,5\},\{2,4\} \succ\{1,5\},\{2,5\} \succ\{3,4\},\{4,5\} \succ\{2\} .
$$


Indeed, the multiset union of the sets on the right and the multiset union of the sets on the left are both equal to the multiset $\left\{1,2^{2}, 3,4^{2}, 5^{2}\right\}$, where powers denote multiplicities. Hence the cancellation condition $C_{4}$ is violated with $a_{1}=a_{2}=a_{3}=a_{4}=1$.

The next proposition follows easily from a result proved by Kraft et al. (1959) for weak comparative probability orderings.

Proposition 5 For a comparative probability ordering to be representable, it is necessary and sufficient that all cancellation conditions $C_{m}$ are satisfied.

These cancellation conditions look rather unnatural and complicated. Since they are derived from linear algebra, it is not surprising that they can be made to look much more natural by being reformulated in terms of vectors, as we do later in this paper.

It is clear that any representable comparative probability ordering satisfies $C_{m}$ for all $m \geq 1$. It was also shown in Fishburn (1996, Section 2) that $C_{2}$ and $C_{3}$ follow from de Finetti's axiom and properties of linear orders. Hence $C_{4}$ is the first nontrivial cancellation condition.

As was noticed in Kraft et al. (1959), for $n<5$ all comparative probability orderings are representable, but for $n=5$ there are non-representable ones. Fishburn (1996, Section 4) showed that all non-representable comparative probability orderings on a 5-element set $X$ fail to satisfy $C_{4}$, and conjectured Fishburn $(1996,1997)$ that any such ordering on an $n$-element set $X$, which satisfies $C_{4}, \ldots, C_{n-1}$, is representable.

In Section 3 we will exhibit counter-examples to this conjecture for $n=7$. More precisely, we will construct non-representable comparative probability orderings on a 7 -element set $X$ which satisfy $C_{4}, C_{5}$ and $C_{6}$. In addition, we will show these orderings are almost representable.

We will always assume here that a linear order $\succeq$ on $2^{X}$ is a comparative probability ordering. As in Fishburn $(1996,1997)$, we will also assume that $1 \succ 2 \succ \ldots \succ n$, which is equivalent to assuming that $p_{1}>p_{2}>\ldots>p_{n}$ for a comparative probability ordering arising from a probability measure $\mathbf{p}=$ $\left(p_{1}, \ldots, p_{n}\right)$. To every such linear order $\succeq$, there corresponds a discrete cone $C(\succeq)$ in $T^{n}$, where $T=\{-1,0,1\}$, as defined in Fishburn (1996).

Definition 6 A subset $C \subseteq T^{n}$ is said to be a discrete cone if the following properties hold:

D1. $\left\{\mathbf{e}_{1}-\mathbf{e}_{2}, \ldots, \mathbf{e}_{n-1}-\mathbf{e}_{n}, \mathbf{e}_{n}\right\} \subseteq C$, where $\left\{\mathbf{e}_{1}, \ldots, \mathbf{e}_{n}\right\}$ is the standard basis of $\mathbb{R}^{n}$, 
D2. $\{-\mathbf{x}, \mathbf{x}\} \cap C \neq \emptyset$ for every $\mathbf{x} \in T^{n}$,

D3. $\mathbf{x}+\mathbf{y} \in C$ whenever $\mathbf{x}, \mathbf{y} \in C$ and $\mathbf{x}+\mathbf{y} \in T^{n}$.

We note that in Fishburn (1996), Fishburn requires $\mathbf{0} \notin C$ because his orders are anti-reflexive. In our case, condition D2 implies $\mathbf{0} \in C$.

Given a (weak) comparative probability ordering $\succeq$ on $2^{X}$, we may construct a characteristic vector $\chi(A, B)=\chi_{A}-\chi_{B} \in T^{n}$ for every possible comparison $A \succeq B$. The cone $C(\succeq)$ is then defined as the set of all characteristic vectors $\chi(A, B)$, for $A, B \in 2^{X}$ such that $A \succeq B$. The two axioms of comparative probability given in Definition 2 guarantee that $C(\succeq)$ is indeed a discrete cone (see Fishburn, 1996, Lemma 2.1).

Example 7 For the comparative probability ordering $\succeq$ from Example 4, the four vectors

$$
(1,-1,1,-1,-1),(-1,1,0,1,-1),(0,1,-1,-1,1),(0,-1,0,1,1)
$$

all belong to $C(\succeq)$, and correspond to the four comparisons listed in (4) above.

We will say that a discrete cone $C \subseteq T^{n}$ is generated by a set of vectors $V=\left\{\mathbf{v}_{1}, \ldots, \mathbf{v}_{m}\right\} \subseteq T^{n}$ if $C$ is the smallest discrete cone containing $V$. It follows that the cone $C(\succeq)$ associated with a comparative probability ordering $\succeq$ must always contains the discrete cone $U$ in $T^{n}$ generated by

$$
\left\{\mathbf{e}_{1}-\mathbf{e}_{2}, \ldots, \mathbf{e}_{n-1}-\mathbf{e}_{n}, \mathbf{e}_{n}\right\}
$$

We observe that a vector $\mathbf{x}=\left(x_{1}, \ldots, x_{n}\right) \in T^{n}$ belongs to $U$ if and only if

$$
x_{1} \geq 0, x_{1}+x_{2} \geq 0, \ldots, x_{1}+x_{2}+\ldots+x_{n} \geq 0 .
$$

Hence, following Fishburn (1996), we can reformulate the cancellation conditions as follows:

Proposition 8 A comparative probability ordering $\succeq$ satisfies the mth cancellation condition $C_{m}$ if and only if for no set $\left\{\mathbf{x}_{1}, \ldots, \mathbf{x}_{m}\right\}$ of non-zero vectors in $C(\succeq)$ do there exist positive integers $a_{1}, \ldots, a_{m}$ such that

$$
a_{1} \mathbf{x}_{1}+a_{2} \mathbf{x}_{2}+\cdots+a_{m} \mathbf{x}_{m}=\mathbf{0} .
$$

PROOF. Take a vector $\mathbf{x}_{i}=\chi\left(A_{i}, B_{i}\right) \in C(\succeq)$ for each comparison $A_{i} \succeq B_{i}$ (for $1 \leq i \leq m$ ) involved in Definition 3, and observe that condition (3) is equivalent to the equation $a_{1} \mathbf{x}_{1}+a_{2} \mathbf{x}_{2}+\cdots+a_{m} \mathbf{x}_{m}=\mathbf{0}$.

Example 9 The four vectors from the cone $C(\succeq)$ given in Example 7 add up to the zero vector, hence the corresponding ordering $\succeq$ is not representable. 
Geometrically, what happens is clear. A comparative probability ordering $\succeq$ is representable if and only if there exists a positive integer-valued vector $\mathbf{a} \in \mathbb{R}^{n}$ such that

$$
\mathbf{x} \in C(\succeq) \Longleftrightarrow(\mathbf{a}, \mathbf{x})>0 \text { for all } \mathbf{x} \in T^{n} \backslash\{\mathbf{0}\}
$$

where $(\cdot, \cdot)$ is the standard inner product; that is, $\succeq$ is representable if and only if every non-zero vector in the cone $C(\succeq)$ lies in the open half-space $S_{\mathbf{a}}=\left\{\mathbf{x} \in \mathbb{R}^{n} \mid(\mathbf{a}, \mathbf{x})>0\right\}$.

Similarly, for a non-representable but almost representable comparative probability ordering $\succeq$, there exists an integer-valued vector $\mathbf{a} \in \mathbb{R}^{n}$ with nonnegative entries such that

$$
\mathbf{x} \in C(\succeq) \Longleftrightarrow(\mathbf{a}, \mathbf{x}) \geq 0 \quad \text { for all } \mathbf{x} \in T^{n} \backslash\{\mathbf{0}\}
$$

and hence in this case the whole cone $C(\succeq)$ must lie in the closed half-space $\overline{S_{\mathbf{a}}}=\left\{\mathbf{x} \in \mathbb{R}^{n} \mid(\mathbf{a}, \mathbf{x}) \geq 0\right\}$.

In both cases, the normalised vector a gives us the probability measure, namely $\mathbf{p}=\left(a_{1}+\ldots+a_{n}\right)^{-1}\left(a_{1}, \ldots, a_{n}\right)$, from which $\succeq$ arises or with which it almost agrees.

\section{Almost representable orderings}

Proposition 10 Let $\succeq$ be a non-representable but almost representable comparative probability ordering which almost agrees with a probability measure $\mathbf{p}$. Suppose that the mth cancellation condition $C_{m}$ is violated, and that for some non-zero vectors $\left\{\mathbf{x}_{1}, \ldots, \mathbf{x}_{m}\right\} \subseteq C(\succeq)$ and some positive integers $a_{1}, \ldots, a_{m}$, the condition (8) holds. Then all of the vectors $\mathbf{x}_{1}, \ldots, \mathbf{x}_{m}$ lie in the hyperplane $H_{\mathbf{p}}=\left\{\mathbf{x} \in \mathbb{R}^{n} \mid(\mathbf{p}, \mathbf{x})=0\right\}$.

PROOF. First note that for every $\mathbf{x} \in C(\succeq)$ which does not belong to $H_{\mathbf{p}}$, we have $(\mathbf{p}, \mathbf{x})>0$. Hence the condition (8) can hold only when all $\mathbf{x}_{i} \in H_{\mathbf{p}}$.

Corollary 11 Any almost representable comparative probability ordering $\succeq$ on an n-element set $X$, which satisfies $C_{4}, \ldots, C_{n}$, is representable.

PROOF. Suppose that (8) holds for some non-zero vectors $\mathbf{x}_{1}, \ldots, \mathbf{x}_{m} \in C(\succeq)$ and positive integers $a_{1}, \ldots, a_{m}$, where $m>0$. As all $m$ vectors $\mathbf{x}_{1}, \ldots, \mathbf{x}_{m}$ lie in the $(n-1)$-dimensional subspace $H_{\mathbf{p}}$, we may, using standard linear algebra, reduce the number of vectors in this linear combination to at most $n$ vectors 
while still having positive coefficients. Hence we may suppose that $m \leq n$. However, this is impossible since we assumed that $C_{m}$ is satisfied. Therefore $\succeq$ must be representable.

Following Fishburn (1996, Section 5), we now define $f(n)$ to be the smallest positive integer $k$ such that every comparative probability ordering on an $n$-element set which satisfies the cancellation conditions $C_{4}, \ldots, C_{k}$ is representable. Fishburn $(1996,1997)$ proved that $f(5)=4$, and $n-1 \leq f(n) \leq n+1$ for all $n \geq 6$, and conjectured that $f(n)=n-1$ for all $n \geq 5$ (see Fishburn, 1997, p.354).

In the spirit of Kraft et al. (1959), we further define $g(n)$ to be the smallest positive integer $k$ such that every almost representable comparative probability ordering on an $n$-element set which satisfies the cancellation conditions $C_{4}, \ldots, C_{k}$ is representable. Clearly $g(n) \leq f(n)$ for all $n$, and also by Corollary 11 above, we have the following:

Corollary $12 \quad g(n) \leq n$ for all $n$.

Now Fishburn's conjecture, if valid, would imply that $g(n) \leq n-1$ for all $n$. We can confirm that $f(6)=5$, which is consistent with Fishburn's conjecture, but we also prove that $g(7)=7$, which refutes it. To do this, we will need the following theorem which simplifies and generalises Theorem 6.1 from Fishburn (1996) (and its generalisation to Lemma 1 in Fishburn (1997)).

Theorem 13 (Construction method 1) Let $X=\left\{\mathbf{x}_{1}, \ldots, \mathbf{x}_{m}\right\}, m \geq 4$, be a system of non-zero vectors from $T^{n}$, such that $\sum_{i=1}^{m} a_{i} \mathbf{x}_{i}=\mathbf{0}$ for some positive integers $a_{1}, \ldots, a_{m}$, and such that no proper subsystem $X^{\prime} \subset X$ is linearly dependent with positive coefficients. Suppose further that the $m \times n$ matrix $A$ having the vectors $\mathbf{x}_{1}, \ldots, \mathbf{x}_{m}$ as its rows has the property that $A \mathbf{b}=$ $\mathbf{0}$ for some positive integer-valued vector $\mathbf{b}=\left(b_{1}, \ldots, b_{n}\right)$ with $b_{1}>b_{2}>\ldots>$ $b_{n}>0$, and that

$$
\operatorname{Span}\left\{\mathbf{x}_{1}, \ldots, \mathbf{x}_{m}\right\} \cap T^{n}=\left\{ \pm \mathbf{x}_{1}, \ldots, \pm \mathbf{x}_{m}\right\}
$$

Let $C(\succeq)$ be the discrete cone belonging to the weak comparative probability ordering which arises from the measure $\mathbf{p}=\left(b_{1}+\ldots+b_{n}\right)^{-1}\left(b_{1}, \ldots, b_{n}\right)$, that is, $C(\succeq)=\left\{\mathbf{x} \in T^{n} \mid(\mathbf{x}, \mathbf{b}) \geq 0\right\}$. Then the discrete cone

$$
C^{\prime}=C(\succeq) \backslash\left\{-\mathbf{x}_{1}, \ldots,-\mathbf{x}_{m}\right\}
$$

corresponds to an almost representable comparative probability ordering which almost agrees with $\mathbf{p}$, and satisfies $C_{i}$ for all $i<m$, but does not satisfy $C_{m}$. 
PROOF. We first note that $\left\{ \pm \mathbf{x}_{1}, \ldots, \pm \mathbf{x}_{m}\right\} \subseteq H_{\mathbf{b}}=\left\{\mathbf{x} \in \mathbb{R}^{n} \mid(\mathbf{b}, \mathbf{x})=0\right\}$. $\operatorname{Next}\left(\mathbf{e}_{i}-\mathbf{e}_{i+1}, \mathbf{b}\right)=b_{i}-b_{i+1}>0$ for $1 \leq i<n$, and also $\left(\mathbf{e}_{n}, \mathbf{b}\right)=b_{n}>0$, so the property D1 holds for the cone $C^{\prime}$. Property D2 holds for $C^{\prime}$ because it holds for $C(\succeq)$ and when we remove vectors $-\mathbf{x}_{1}, \ldots,-\mathbf{x}_{m}$ from $C^{\prime}$ the vectors $\mathbf{x}_{1}, \ldots, \mathbf{x}_{m}$ remain in $C^{\prime}$. Finally let us prove D3. Suppose that $\mathbf{y}, \mathbf{z} \in C^{\prime}$ and $\mathbf{y}+\mathbf{z} \in T^{n}$, but $\mathbf{y}+\mathbf{z} \notin C^{\prime}$. Since $\mathbf{y}+\mathbf{z} \in C(\succeq) \backslash C^{\prime}$, we deduce that $\mathbf{y}+\mathbf{z}=-\mathbf{x}_{i}$ for some $i$. Moreover, $\mathbf{y}, \mathbf{z} \in H_{\mathbf{b}}$, since otherwise $(\mathbf{y}+\mathbf{z}, \mathbf{b})=(\mathbf{y}, \mathbf{b})+(\mathbf{z}, \mathbf{b})>0$. Hence $\mathbf{y}=\mathbf{x}_{j}$ for some $j$ and $\mathbf{z}=\mathbf{x}_{k}$ for some $k$, but from this it follows that $\mathbf{x}_{i}+\mathbf{x}_{j}+\mathbf{x}_{k}=\mathbf{0}$, a contradiction since $|X|=m \geq 4$ and hence no three rows of $A$ may have sum zero.

Suppose now that some violation of $C_{k}$ occurs, say

$$
c_{1} \mathbf{y}_{1}+c_{2} \mathbf{y}_{2}+\cdots+c_{k} \mathbf{y}_{k}=\mathbf{0}
$$

with positive integers $c_{1}, \ldots, c_{k}$ and $\mathbf{y}_{i} \in C^{\prime}$ for $1 \leq i \leq k$, where $k<m$. If $\left(\mathbf{y}_{i}, \mathbf{b}\right)>0$ from some $i$, then $(\mathbf{0}, \mathbf{b})=\left(c_{1} \mathbf{y}_{1}+c_{2} \mathbf{y}_{2}+\cdots+c_{k} \mathbf{y}_{k}, \mathbf{b}\right)=$ $c_{1}\left(\mathbf{y}_{1}, \mathbf{b}\right)+c_{2}\left(\mathbf{y}_{2}, \mathbf{b}\right)+\ldots+c_{k}\left(\mathbf{y}_{k}, \mathbf{b}\right)>0$, a contradiction, hence $\mathbf{y}_{i} \in H_{\mathbf{b}}$ for all $i$, and therefore $\left\{\mathbf{y}_{1}, \ldots, \mathbf{y}_{k}\right\} \subseteq\left\{\mathbf{x}_{1}, \ldots, \mathbf{x}_{m}\right\}$, which is impossible. On the other hand, $C_{m}$ fails because $\sum_{i=1}^{m} a_{i} \mathbf{x}_{i}=\mathbf{0}$.

A major difference between our theorem and Theorem 6.1 of Fishburn (1996) is that we claim almost representability of the constructed ordering. Using this, we can now prove the following:

Theorem $14 \quad f(7) \geq g(7)=7$.

PROOF. Consider the following $7 \times 7$ matrix

$$
A=\left[\begin{array}{rrrrrrr}
-1 & 1 & 1 & -1 & 0 & 0 & 0 \\
1 & 0 & -1 & -1 & 1 & 0 & 0 \\
1 & -1 & 1 & 0 & 0 & -1 & -1 \\
-1 & -1 & 0 & 1 & 1 & 0 & 1 \\
-1 & 1 & -1 & 1 & 0 & 1 & -1 \\
1 & -1 & 1 & -1 & -1 & 1 & 0 \\
0 & 1 & -1 & 1 & -1 & -1 & 1
\end{array}\right] .
$$

Denote the rows of $A$ by $\mathbf{x}_{1}, \ldots, \mathbf{x}_{7}$. As the null space of $A^{T}$ is spanned by the vector $(1,1,1,1,1,1,1)$, we can see that $\mathbf{x}_{1}+\mathbf{x}_{2}+\ldots+\mathbf{x}_{7}=\mathbf{0}$ and that no six rows of $A$ are linearly dependent. We also observe that $A \mathbf{b}=$ 
$\mathbf{0}$ for $\mathbf{b}=(27,25,24,22,19,15,11)$. Let us take the probability measure $\mathbf{p}=\frac{1}{116}(27,25,24,22,19,15,11)$, and now consider the weak comparative probability ordering on $I_{7}=\{1,2,3,4,5,6,7\}$ that arises from $\mathbf{p}$.

Ignoring braces when writing subsets, and listing only half of all terms (as $A \succeq B$ if and only if $\bar{B} \succeq \bar{A}$ ), we may describe this ordering by the following sequence (given in reverse order for simplicity):

$\emptyset \prec 7 \prec 6 \prec 5 \prec 4 \prec 3 \prec 2 \prec 67 \prec 1 \prec 57 \prec 47 \prec 56 \prec 37 \prec 27 \prec 46 \prec 17 \prec$ $36 \prec 26 \prec 45 \prec 16 \prec 35 \prec 25 \prec 567 \prec 15 \sim 34 \prec 24 \prec 467 \prec 23 \sim 14 \prec$ $367 \prec 13 \sim 267 \prec 457 \sim 12 \prec 167 \prec 357 \prec 257 \prec 456 \prec 157 \sim 347 \prec 247 \sim$ $356 \prec 256 \prec 237 \sim 147 \prec 156 \sim 346 \prec 246 \sim 137 \prec 127 \prec 236 \sim 146 \prec$ $345 \prec 136 \sim 245 \prec 4567 \sim 126 \prec 235 \sim 145 \prec 3567 \prec 135 \sim 2567 \prec 125 \sim$ $234 \prec \ldots$.

The only equivalences in the first half of this sequence are these:

$$
\begin{aligned}
& 15 \sim 34, \quad 157 \sim 347, \quad 156 \sim 346, \quad 125 \sim 234 \\
& 23 \sim 14, \quad 237 \sim 147, \quad 236 \sim 146, \quad 235 \sim 145 \\
& 13 \sim 267, \quad 135 \sim 2567, \\
& 457 \sim 12, \quad 4567 \sim 126, \\
& 246 \sim 137 \\
& 136 \sim 245 \\
& 247 \sim 356 .
\end{aligned}
$$

Note that in each row of the above list, all equivalences are the consequences of the leftmost equivalence, and all contribute the same pair of vectors $\pm \mathbf{x}_{i}$ to the cone $C(\succeq)$, where $\mathbf{x}_{1}, \ldots, \mathbf{x}_{7}$ are the rows of $A$. All equivalences from the second half of the sequence are also consequences of these. It follows that

$$
\operatorname{Span}\left\{\mathbf{x}_{1}, \ldots, \mathbf{x}_{7}\right\} \cap T^{7}=\left\{ \pm \mathbf{x}_{1}, \ldots, \pm \mathbf{x}_{7}\right\}
$$

We may now apply Theorem 13, to deduce the existence of a non-representable comparative probability ordering on $I_{7}$, which satisfies $C_{4}, C_{5}$ and $C_{6}$.

Note that the example given in the above proof fails the cancellation condition $C_{7}$ with $\left(a_{1}, \ldots, a_{7}\right)=(1,1,1,1,1,1,1)$. There are hundreds of other examples with the same property. Another interesting example will be given in Section 4 . 


\section{Alternative form of cancellation conditions}

In the spirit of Fishburn's earlier paper (Fishburn, 1996), we now introduce an alternative form of cancellation conditions.

Definition 15 A comparative probability ordering $\succeq$ is said to satisfy the mth cancellation condition $C_{m}^{\dagger}$ if for no set $\left\{\mathbf{x}_{1}, \ldots, \mathbf{x}_{k}\right\}$ of non-zero vectors in $C(\succeq)$ do there exist positive integers $a_{1}, \ldots, a_{k}$ such that

$$
a_{1} \mathbf{x}_{1}+a_{2} \mathbf{x}_{2}+\cdots+a_{k} \mathbf{x}_{k}=\mathbf{0}
$$

and $\sum_{i=1}^{k} a_{i}=m$.

Note here that while the condition $C_{m}$ emphasises the total number of distinct comparisons $A_{i} \succ B_{i}$ involved, the alternative condition $C_{m}^{\dagger}$ is more concerned with the total number of (not necessarily distinct) comparisons actually required for the sum.

It is clear (and was observed by Fishburn (1996, Section 2)) that a comparative probability ordering satisfies $C_{m}$ for all $m \geq 4$ if and only if it satisfies $C_{m}^{\dagger}$ for all $m \geq 4$. It follows that these alternative cancellation conditions can also be used for measuring the degree of deviation of a comparative probability ordering from one that arises from a probability measure.

Hence we may define $f^{\dagger}(n)$ to be the smallest positive integer $k$ such that every comparative probability ordering on an $n$-element set, which satisfies the cancellation conditions $C_{4}^{\dagger}, \ldots, C_{k}^{\dagger}$, is representable. The corresponding analogue of $g(n)$ can also be introduced. We define $g^{\dagger}(n)$ to be the smallest positive integer $k$ such that every almost representable comparative probability ordering on an $n$-element set, which satisfies the cancellation conditions $C_{4}^{\dagger}, \ldots, C_{k}^{\dagger}$, is representable. Obviously, $f^{\dagger}(n) \geq g^{\dagger}(n)$, and also $f^{\dagger}(n) \geq f(n)$ and $g^{\dagger}(n) \geq g(n)$ for all $n$. Also Kraft et al. (1959) effectively commented without proof that $f^{\dagger}(n) \leq n$ ! (see Kraft et al., 1959, p. 413).

Despite such a large upper bound, it appears that to date no examples have been found for which $f^{\dagger}(n) \neq f(n)$ or $g^{\dagger}(n) \neq g(n)$. We show below that $f^{\dagger}(7) \geq g^{\dagger}(7) \geq 8$, which, together with our earlier finding that $g(7)=7$, proves that $g^{\dagger}(7)>g(7)$. To do this, we will need a variation of Theorem 13 .

Theorem 16 (Construction method 2) Let $X=\left\{\mathbf{x}_{1}, \ldots, \mathbf{x}_{k}\right\}, k \geq 4$, be a system of non-zero vectors from $T^{n}$ such that $\sum_{i=1}^{k} a_{i} \mathbf{x}_{i}=0$ for some non-negative integers $a_{1}, \ldots, a_{k}$ with $\sum_{i=1}^{k} a_{i}=m$, and such that for every equation $\sum_{i=1}^{k} a_{i}^{\prime} \mathbf{x}_{i}=\mathbf{0}$ with positive integer coefficients $a_{i}^{\prime}$ it happens that $\sum_{i=1}^{k} a_{i}^{\prime} \geq m$. Suppose further that the $k \times n$ matrix $A$ having the vectors $\mathbf{x}_{1}, \ldots, \mathbf{x}_{k}$ as its rows has the property that $A \mathbf{b}=\mathbf{0}$ for some positive integer- 
valued vector $\mathbf{b}=\left(b_{1}, \ldots, b_{n}\right)$ with $b_{1}>b_{2}>\ldots>b_{n}>0$, and that

$$
\operatorname{Span}\left\{\mathbf{x}_{1}, \ldots, \mathbf{x}_{k}\right\} \cap T^{n}=\left\{ \pm \mathbf{x}_{1}, \ldots, \pm \mathbf{x}_{k}\right\} .
$$

Let $C(\succeq)$ be the discrete cone belonging to the weak comparative probability ordering which arises from the measure $\mathbf{p}=\left(b_{1}+\ldots+b_{n}\right)^{-1}\left(b_{1}, \ldots, b_{n}\right)$, that is, $C(\succeq)=\left\{\mathbf{x} \in T^{n} \mid(\mathbf{x}, \mathbf{b}) \geq 0\right\}$. Then the discrete cone

$$
C^{\prime}=C(\succeq) \backslash\left\{-\mathbf{x}_{1}, \ldots,-\mathbf{x}_{m}\right\}
$$

corresponds to an almost representable comparative probability ordering which almost agrees with $\mathbf{p}$, and satisfies $C_{i}^{\dagger}$ for all $i<m$, but does not satisfy $C_{m}^{\dagger}$.

PROOF. The proof is completely analogous to the proof of Theorem 13 .

Theorem $17 \quad f^{\dagger}(7) \geq g^{\dagger}(7) \geq 8$.

PROOF. Consider the following $7 \times 7$ matrix:

$$
B=\left[\begin{array}{rrrrrrr}
1 & -1 & -1 & 1 & 0 & 1 & -1 \\
1 & 0 & -1 & -1 & 1 & -1 & -1 \\
1 & 0 & -1 & -1 & -1 & 0 & 1 \\
-1 & 1 & -1 & 1 & 1 & 0 & 1 \\
0 & -1 & 1 & 1 & 0 & -1 & 1 \\
0 & -1 & 1 & -1 & 1 & 1 & 1 \\
-1 & 1 & 1 & 0 & -1 & 0 & -1
\end{array}\right],
$$

and let $\mathbf{x}_{1}, \ldots, \mathbf{x}_{7}$ denote its rows. It is easy to check that

$$
\mathbf{x}_{1}+\ldots+\mathbf{x}_{6}+2 \mathbf{x}_{7}=\mathbf{0}
$$

that is, condition $C_{7}$ is violated with $\left(a_{1}, \ldots, a_{7}\right)=(1,1,1,1,1,1,2)$, and there does not exist any "smaller" violation. Calculations also show that

$$
\operatorname{Span}\left\{\mathbf{x}_{1}, \ldots, \mathbf{x}_{7}\right\} \cap T^{7}=\left\{ \pm \mathbf{x}_{1}, \ldots, \pm \mathbf{x}_{7}\right\}
$$

and $A \mathbf{p}=\mathbf{0}$ for the probability measure $\mathbf{p}=\frac{1}{148}(48,40,27,16,12,10,7)$. For the weak comparative probability ordering determined by this measure, the equivalences are all consequences of one of the following seven: $146 \sim 237$, $15 \sim 3467,17 \sim 345,2457 \sim 13,347 \sim 26,3567 \sim 24$ and $23 \sim 157$. Again 
this is a non-representable but almost representable comparative probability ordering on $I_{7}$, but is one which satisfies $C_{4}^{\dagger}, C_{5}^{\dagger}, C_{6}^{\dagger}$, and $C_{7}^{\dagger}$, while failing $C_{8}^{\dagger}$ with $\left(a_{1}, \ldots, a_{7}\right)=(1,1,1,1,1,1,2)$.

\section{Account for $\mathrm{n}=5$ and $\mathrm{n}=6$}

The results of this section were obtained with the help of the computer algebra system MaGma (Bosma et al., 1997), using the same techniques of enumeration as described in Fishburn (1996, Section 4), but also checking the results for both representability and almost representability (using techniques of linear programming). Enumeration of the orderings required less than 60 seconds of computer time for the case $n=5$, and 12 hours for the case $n=6$, while determining representability (and/or almost representability) took just a little longer.

Theorem 18 There are 546 different comparative probability orderings of the subsets of $I_{5}=\{1,2,3,4,5\}$, of which 516 are representable, and the remaining 30 are almost representable.

In particular, our computations show that Fishburn's claimed total number of 561 orderings in Fishburn (1996, Section 4) is incorrect.

Note also that it is quite easy to decide if any given one of these orderings is almost representable. For example, one of the 30 non-representable orderings (the last in the list at the end of Section 4 of Fishburn (1996)) is completely determined by the four mutually-cancelling comparisons $234 \succeq 15,1 \succeq 245$, $25 \succeq 34,45 \succeq 2$, and the extra conditions $235 \succ 1$ and $1 \succ 23$. Now if this ordering arises from the probability measure $\mathbf{p}=\left(p_{1}, \ldots, p_{5}\right)$, then the first four comparisons require that $p_{1}=2 p_{4}+2 p_{5}, p_{2}=p_{4}+p_{5}$ and $p_{3}=2 p_{5}$, and the two extra conditions require that $2 p_{5}>p_{4}$ and $p_{4}>p_{5}$. Choosing $p_{4}=3$ and $p_{5}=2$, for instance, we see that the given ordering almost agrees with the probability measure $\mathbf{p}=\frac{1}{24}(10,5,4,3,2)$.

Theorem 19 There are exactly 169444 different comparative probability orderings of the subsets of $I_{6}=\{1,2,3,4,5,6\}$. Of these, 124187 are representable, while 45257 are not. Of the 45257 that are non-representable, 44987 fail $C_{4}$ with $a_{1}=a_{2}=a_{3}=a_{4}=1$, while the other 270 satisfy $C_{4}$ but fail $C_{5}$ with $a_{1}=a_{2}=a_{3}=a_{4}=a_{5}=1$. Moreover, of these 45257 orderings that are non-representable, 40055 are almost representable, while the other 5202 are not. In particular, of the 44987 non-representable orderings that fail $C_{4}$, exactly 39785 are almost representable, while the other 5202 are not, and all of the 270 non-representable orderings that satisfy $C_{4}$ but fail $C_{5}$ are almost representable. Hence there are exactly 164242 almost representable orderings, 
and of these, 124187 are representable, while 40055 are not, with 39785 failing $C_{4}$ with $a_{1}=a_{2}=a_{3}=a_{4}=1$, and the other 270 satisfying $C_{4}$ but failing $C_{5}$ with $a_{1}=a_{2}=a_{3}=a_{4}=a_{5}=1$.

We say that the dual of a comparison $A \succ B$ is the comparison $B \succ A$, and then the dual of a set of comparisons is the set of their duals. In the case $n=5$, Fishburn categorised the 30 orderings that are non-representable into five types (and their duals) according to the violations of $C_{4}$ that they exhibit, and noted that some orderings may exhibit more than one type of violation. For the case $n=6$, we find that up to duality there are 423 such types, of which 385 (and their duals) involve four comparisons that together fail $C_{4}$ with $a_{1}=a_{2}=a_{3}=a_{4}=1$, while the remaining 38 (and their duals) involve five comparisons that together fail $C_{5}$ with $a_{1}=a_{2}=a_{3}=a_{4}=a_{5}=1$.

We note that the first example of a comparative probability ordering on a 6 -element set which fails to be almost representable was constructed by Kraft et al. (1959). Our computations provide the following extension to the results of Fishburn (1996, 1997):

Corollary $20 \quad f(6)=g(6)=f^{\dagger}(6)=g^{\dagger}(6)=5$.

\section{Conclusion and Open Questions}

Kraft, Pratt, and Seidenberg (Kraft et al., 1959) left open the question of whether or not all comparative probability orderings on a 5-element set are almost representable. We have answered this question by showing that all of them are indeed almost representable.

We know now that $f(5)=g(5)=4$, and $f(6)=g(6)=5$, but $g(7)=7$. The question remains open as to whether $f(7)$ is equal to 7 or 8 . Nevertheless, it is clear that the situation for $n \geq 7$ is different to that for $n \in\{5,6\}$, so Fishburn's conjecture is no longer adequate. We propose amending it to the following:

Conjecture $21 \quad f(n)=g(n)=n$ for all $n \geq 7$.

Some more research will have to be undertaken before any reasonable conjecture about $f^{\dagger}(n)$ and $g^{\dagger}(n)$ can be formulated. 


\section{References}

[1] Bosma, W., Cannon, J., and Playoust, C. (1997). The Magma Algebra System I: The User Language, J. Symbolic Comput. 24, 235-265.

[2] Danilov, V.I. (1987). Aggregation of dichotomic preferences, Mathematical Social Sciences 13, 49-58.

[3] de Finnetti, B. (1931). Sul significato soggetivo della probabilità, Fundamenta Mathematicae 17, 298-329.

[4] Fishburn, P.C. (1996). Finite Linear Qualitative Probability, Journal of Mathematical Psychology 40, 64-77.

[5] Fishburn, P.C. (1997). Failure of Cancellation Conditions for Additive Linear Orders, Journal of Combinatorial Designs 5, 353-365.

[6] Kraft, C.H., Pratt, J.W., and Seidenberg, A. (1959). Intuitive Probability on Finite Sets, Annals of Mathematical Statistics 30, 408-419.

[7] Krantz, D.H., Luce, R.D., Suppes, P., and Tversky, A. (1971). Foundations of Measurement (Vol. 1). Academic Press.

[8] Luce, R.D., and Suppes, P. (1965). Preference, Utility, and Subjective Probability. In: Luce, R.D., Bush, R.R., Galanter, E. (Eds.) Handbook of Mathematical Psychology, Vol. III ( pp. 249-410). Wiley.

[9] Regoli, G.(2000). Comparative probability orderings, preprint on the Imprecise Probabilities Project website http://ippserv.rug.ac.be.

[10] Savage, L.J. (1954). The Foundations of Statistics. New York: Wiley.

[11] Scott, D. (1964) Measurement structures and inequalities, Journal of Mathematical Psychology 1, 233-247.

[12] Stanley, R.P. (1997). Enumerative Combinatorics (Vol.1). Cambridge University Press.

[13] Suppes, P. (1974). Measurement of Belief, Journal of the Royal Statistical Society Series B, 36, 160-191. 